\title{
Estimação dos Parâmetros do Modelo para Predizer Runtime e Características V-I de uma Bateria utilizando Algoritmo Genético
}

\author{
Marcia de Fatima Brondani ${ }^{1}$ \\ Airam Teresa Zago Romcy Sausen² \\ Paulo Sérgio Sausen ${ }^{3}$ \\ Manuel Osório Binelo ${ }^{4}$ \\ Departamento de Ciências Exatas e Engenharia, Programa de Pós-Graduação Stricto Sensu em \\ Modelagem Matemática, Unijuí, Ijuí, RS
}

\begin{abstract}
Resumo. Neste trabalho é proposta uma metodologia baseada em Algoritmo Genético (AG) para a estimação dos parâmetros do modelo para Predizer Runtime e Características V-I, sendo este utilizado para a modelagem matemática do tempo de vida de baterias de Lítio Íon Polímero (LiPo). A validação do modelo e do método utilizado para a sua parametrização é realizada por meio de uma análise comparativa entre os resultados simulados e os dados experimentais, coletados em uma plataforma de testes. Os resultados obtidos demonstram a acurácia do modelo em predizer o tempo de vida das baterias aqui estudadas, além do bom desempenho do método heurístico AG aplicado ao processo de parametrização.
\end{abstract}

Palavras-chave. Estimação de Parâmetros, Tempo de Vida de Baterias, Algoritmos Genéticos, Modelo Elétrico

\section{Introdução}

A demanda por dispositivos móveis mais eficientes tem impulsionado avanços em tecnologias de baterias recarregáveis. As baterias de Lítio Íon Polímero (LiPo) apresentam alta densidade de energia e de potência, sendo mais leves e mais compactas [5]. No entanto, possuem uma vida útil de aproximadamente 400 ciclos de carga/descarga, o que resulta na substituição mais frequente, implicando assim um maior desperdício de energia por meio da produção e do transporte, bem como, um aumento no descarte [2].

Neste contexto, a predição do tempo de vida de baterias a partir de modelos matemáticos $[1,4,6,7]$ pode contribuir para a otimização do uso e projeto de novas baterias, incorrendo no desenvolvimento de tecnologias de energia sustentável. Dentre os modelos aplicados para esta finalidade, destaca-se o modelo elétrico para Predizer Runtime

\footnotetext{
${ }^{1}$ brondani.marcia@gmail.com

2airam@unijui.edu.br

${ }^{3}$ sausen@unijui.edu.br

${ }^{4}$ manuel.binelo@unijui.edu.br
} 
e Características V-I [1]. Este modelo é capaz de capturar as características elétricas e dinâmicas, tais como, a tensão em circuito aberto, a capacidade utilizável e a resposta transiente [1]. Entretanto, os parâmetros deste modelo são usualmente obtidos por meio de experimentação extensiva, o que torna esta parametrização relativamente empírica [1,4].

Neste trabalho é proposta uma metodologia baseada em Algoritmo Genético (AG) [3] para a estimação dos parâmetros do modelo para Predizer Runtime e Características V-I, aplicado à modelagem matemática do tempo de vida de baterias de LiPo. O AG proposto e o modelo são implementados no software Matlab. A validação do modelo e do método utilizado para a sua parametrização é realizada por meio de uma análise comparativa entre os resultados simulados e os dados experimentais, coletados em uma plataforma de testes.

O restante deste artigo está organizado como segue. Na Seção 2 é apresentado o modelo para Predizer Runtime e Características V-I, bem como são descritas suas equações. $\mathrm{Na}$ Seção 3 são apresentados os dados experimentais utilizados para a estimação dos parâmetros e para a validação do modelo. Na Seção 4 é descrita a metodologia proposta para a estimação dos parâmetros baseada em AG. Na Seção 5 são apresentados os resultados das simulações computacionais. E, na Seção 6 é apresentada a conclusão.

\section{Modelo para Predizer Runtime e Características V-I}

O modelo para Predizer Runtime e Características V-I [1] foi proposto a partir da combinação de modelos baseados em Runtime (circuito da esquerda) e em Thevenin (circuito da direita), conforme apresentado na Figura 1. O circuito da esquerda é composto

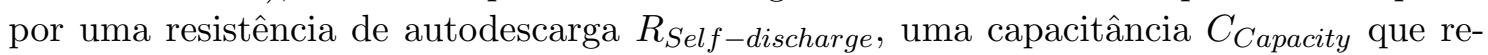
presenta a carga total armazenada na bateria e uma fonte de corrente controlada $I_{B a t t}$. Estes são aplicados para modelar a capacidade, o estado de carga $S O C$ e o tempo de vida da bateria. Já o circuito da direita fornece a resposta transiente, sendo constituído por uma resistência $R_{\text {Series }}$, duas redes resistivas capacitivas $R C$ dispostas em paralelo e uma fonte de tensão controlada $V_{O C}\left(V_{S O C}\right)$. Esta última representa a relação não linear entre a tensão de circuito aberto $V_{O C}$ e o $S O C$, descrito pela tensão $V_{S O C}$ [1].

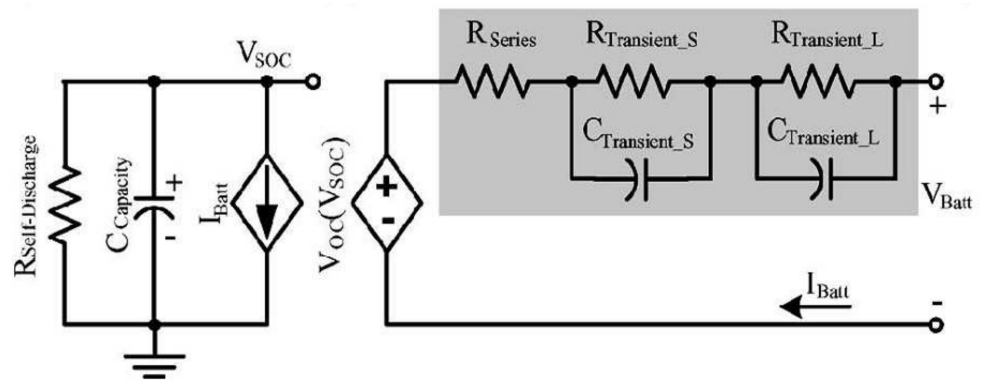

Figura 1: Modelo para Predizer Runtime e Características V-I [1].

Para modelar a resposta transiente são consideradas duas constantes de tempo, de curta e longa duração. Estas são definidas no período de $t_{0}<t<t_{r}$, em que $t_{0}$ é o tempo inicial e $t_{r}$ é o tempo de término do período. Durante o intervalo de $t_{0}<t<t_{d}$, a bateria 
é descarregada com uma corrente constante $\left(I_{B a t t}>0\right)$ e, de $t_{d}<t<t_{r}$, ela permanece ociosa $\left(I_{\text {Batt }}=0\right)$, sendo $t_{d}$ o tempo final da descarga [4]. As respostas transientes de curta e de longa duração são representadas pelas seguintes resistências e capacitâncias, respectivamente: $R_{\text {Transient_S }}, C_{\text {Transient_S }}, R_{\text {Transient_L }}$ e $C_{\text {Transient_L }}$. Em estado estacionário, $C_{\text {Transient_S }}$ e $C_{\text {Transient_L }}$ desempenham a função de um circuito aberto, oferecendo uma alta resistência à corrente contínua. No entanto, em condição transiente, estes capacitores se comportam como um curto circuito para o momento transitório, até que estejam totalmente carregados [1].

A capacidade utilizável da bateria é capturada a partir da relação estabelecida entre os componentes $C_{\text {Capacity }}, R_{\text {Self-discharge }}, R_{\text {Series }}, R_{\text {Transient_S }}$ e $R_{\text {Transient_L. }}$ O cálculo da $C_{\text {Capacity }}$ é dado pela equação

$$
C_{\text {Capacity }}=3600 \text { Capacity }_{1}(\text { Cycle }) f_{2}(\text { Temp }),
$$

onde: Capacity é a capacidade nominal, $f_{1}($ Cycle) é o fator de correção do número de ciclos e $f_{2}(T e m p)$ é o fator de correção da temperatura.

A variação da capacidade utilizável é causada pela intensidade da corrente de descarga aplicada à bateria, sendo negligenciados outros aspectos que também podem alterá-la, tais como o número de ciclos, a temperatura e o tempo de armazenamento. Logo, o valor da

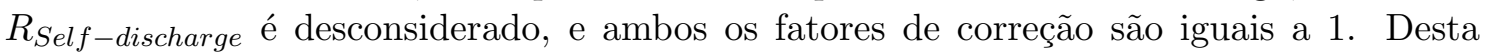
forma, diferentes correntes aplicadas a $C_{\text {Capacity }}$ implicam as quedas de tensão ao longo do resistor equivalente, que corresponde à soma das resistências $R_{\text {Series }}, R_{\text {Transient_S }}$ e $R_{\text {Transient_L }}$. Isto resulta em um conjunto de valores de $S O C$ medidos distintamente de acordo com o decaimento da tensão, diminuindo gradativamente a capacidade utilizável da bateria durante a sua descarga [1].

Assim, a tensão de saída $V_{\text {Batt }}$ pode ser expressa como

$$
V_{\text {Batt }}=V_{O C}\left(V_{S O C}\right)-I_{\text {Batt }} R_{\text {Series }}-V_{\text {Transient }}(t),
$$

onde: $V_{\text {Transient }}(t)$ é a tensão transiente, calculada a partir da equação

$$
V_{\text {Transient }}(t)=V_{\text {Transient_S }}(t)+V_{\text {Transient_L }}(t),
$$

onde: $V_{\text {Transient_S }}(t)$ é a tensão transiente de curta duração e $V_{\text {Transient_L }}(t)$ é a tensão transiente de longa duração, ambas dadas pelas equações (4) e (5), respectivamente,

$$
\begin{aligned}
& V_{\text {Transient_S }}(t)= \begin{cases}R_{\text {Transient_S }} I_{\text {Batt }}(t)\left[1-e^{\left.-\frac{\left(t-t_{0}\right)}{\tau_{S}}\right],}\right. & t_{0}<t<t_{d} \\
V_{\text {Transient_S }}\left(t_{d}\right) e^{-\frac{\left(t-t_{d}\right)}{\tau_{S}}}, & t_{d}<t<t_{r}\end{cases} \\
& V_{\text {Transient_L }}(t)= \begin{cases}R_{\text {Transient_L } L} I_{\text {Batt }}(t)\left[1-e^{\left.-\frac{\left(t-t_{0}\right)}{\tau_{L}}\right],},\right. & t_{0}<t<t_{d} \\
V_{\text {Transient_L } L}\left(t_{d}\right) e^{-\frac{\left(t-t_{d}\right)}{\tau_{L}}}, & t_{d}<t<t_{r}\end{cases}
\end{aligned}
$$


onde: $V_{\text {Transient_S }}\left(t_{d}\right)$ é a tensão transiente de curta duração no tempo final da descarga, $\tau_{S}=R_{\text {Transient_S }} C_{\text {Transient_S }}, V_{\text {Transient_L }}\left(t_{d}\right)$ é a tensão transiente de longa duração no tempo final da descarga e $\tau_{L}=R_{\text {Transient_L }} C_{\text {Transient_L }}$ [4].

Os elementos $V_{O C}$ e $R_{\text {Series }}$ da equação (2), assim como $R_{\text {Transient_S }}, C_{\text {Transient_S }}$, $R_{\text {Transient_L }}$ e $C_{\text {Transient_L }}$, que modelam a resposta transiente de tensão, são funções de $S O C$, descritas pelas seguintes equações

$$
\begin{gathered}
V_{O C}(S O C)=a_{0} e^{-a_{1} S O C}+a_{2}+a_{3} S O C-a_{4} S O C^{2}+a_{5} S O C^{3}, \\
R_{\text {Series }}(S O C)=b_{0} e^{-b_{1} S O C}+b_{2}, \\
\\
\left\{\begin{array}{l}
R_{\text {transient_S }}(S O C)=c_{0} e^{-c_{1} S O C}+c_{2}, \\
C_{\text {transient_S }}(S O C)=d_{0} e^{-d_{1} S O C}+d_{2}, \\
R_{\text {transient_L }}(S O C)=e_{0} e^{-e_{1} S O C}+e_{2}, \\
C_{\text {transient_L }}(S O C)=f_{0} e^{-f_{1} S O C}+f_{2} .
\end{array}\right.
\end{gathered}
$$

A metodologia adotada na literatura para a estimação dos 21 parâmetros das equações (6), (7) e (8) é baseada na técnica de ajuste de curvas, utilizando 4 curvas pulsadas de descargas de baterias [1]. Neste trabalho é proposto um outro método de estimar estes parâmetros a partir da aplicação de AG, tendo como referência uma única curva pulsada de descarga. Esta curva é gerada por meio de dados experimentais, apresentados a seguir.

\section{Dados experimentais}

Neste trabalho são utilizados dados experimentais para a estimação dos parâmetros do modelo para Predizer Runtime e Características V-I, da mesma forma que para a validação do mesmo. Estes dados são referentes às baterias de LiPo novas, modelo PL383562 - 2C, coletados a partir de uma plataforma de testes desenvolvida pelo Grupo de Automação Industrial e Controle (GAIC), da Unijuí.

Os testes experimentais realizados para a parametrização do modelo são baseados no procedimento adotado por [1], que considera uma curva de descarga pulsada contendo de 6 a 10 pulsos. Desta forma, para este estudo é utilizada a curva de descarga pulsada de $400 \mathrm{~mA}$, com o período de $1300 \mathrm{~s}$, em que nos primeiros $700 \mathrm{~s}$ é aplicada a corrente de descarga, e nos $600 s$ restantes a bateria permanece ociosa. Esta sequência é repetida até a bateria atingir a tensão de Cutoff correspondente a $3 \mathrm{~V}$. Destaca-se que esta curva é escolhida a partir de 6 ensaios realizados com diferentes baterias, os quais apresentam comportamentos muito semelhantes e as curvas geradas ficam muito próximas.

Para a validação do modelo é considerado um conjunto de perfis de descargas constantes, que abrange correntes baixas, médias e altas, respeitando a capacidade de $800 \mathrm{~mA}$, limitada pelas baterias aqui estudadas. Logo, são definidos 4 perfis: $100 \mathrm{~mA}, 250 \mathrm{~mA}, 500$ $m A$ e $650 m A$. Para cada um destes perfis são realizados 6 ensaios, obtendo o tempo de vida experimental $\left(t_{v e}\right)$ de cada ensaio e o $t_{v e}$ médio do perfil. Este último é usado como métrica para o cálculo do erro do modelo. Estas baterias também são descarregadas até atingirem a tensão de $3 \mathrm{~V}$. 


\section{Estimação de parâmetros}

A estimação dos parâmetros do modelo para Predizer Runtime e Características V-I é realizada a partir da aplicação do método heurístico AG [3]. Considera-se, na proposição deste algoritmo, a interação entre os dados experimentais e os resultados simulados pelo modelo análoga ao meio ambiente. O código genético de cada indivíduo corresponde aos 21 parâmetros do modelo que necessitam ser estimados, apresentados nas equações (6)-(8). Cada parâmetro é um cromossomo, discretizado em quatro dígitos decimais, que são os genes. A aptidão de cada parâmetro está diretamente relacionada à função objetivo $F$, que caracteriza este problema de otimização, dada pela equação

$$
F=f_{c}\left(\int_{0}^{t_{v e}}\left|V_{\text {Batt }}-V_{e}\right| d t\right)+f_{v}\left(\left|t_{v s}-t_{v e}\right|\right),
$$

onde: $f_{c}$ é o fator de importância da curva experimental, $t_{v e}$ é o tempo de vida experimental $(s), V_{B a t t}$ é a tensão simulada pelo modelo $(V), V_{e}$ é a tensão experimental $(V), f_{v}$ é o fator de importância do tempo de vida experimental e $t_{v s}$ é o tempo de vida simulado pelo modelo $(s)$.

Desta forma, o AG é implementado no software Matlab e é inicializado com uma população aleatória, definida de acordo com os limites mínimos e máximos estabelecidos para cada parâmetro. A cada iteração do AG, os valores dos parâmetros otimizados são informados ao modelo e este é executado, fornecendo a curva simulada que descreve o decaimento da tensão em função do tempo e consequentemente, o tempo de vida simulado ao atingir o nível de Cutoff. Além dos dados simulados, é utilizada como referência a curva experimental pulsada de $400 \mathrm{~mA}$, com o respectivo tempo de vida experimental.

A função aptidão é fundamentada no método de mapeamento Escala de Potência, sendo obtida a partir do inverso de $F$ (equação (9)), ou seja, $F^{-1}$. Isto significa que quanto menor é o valor de $F$, maior é a aptidão dos parâmetros para o caso estudado. Assim, ao atingir o critério de parada, o AG fornece os valores dos 21 parâmetros do modelo para Predizer Runtime e Características V-I otimizados, contidos no indivíduo mais apto. Ressalta-se que a configuração do AG se dá de maneira empiríca por meio da realização de vários testes, considerando aspectos fundamentais tais como a estabilidade, a convergência, a qualidade das respostas e os limites impostos pelo custo computacional do algoritmo.

Com a definição dos parâmetros, o modelo é testado para diferentes perfis de descargas, incluindo o perfil de descarga utilizado para a estimação dos seus parâmetros. Este procedimento é realizado a fim de validar a metodologia proposta para a estimação dos parâmetros, bem como verificar a acurácia do modelo ao aplicá-lo para simular o tempo de vida de baterias de LiPo.

\section{Resultados}

Nesta seção são apresentados os principais resultados das simulações realizadas com o modelo para Predizer Runtime e Características V-I utilizando os parâmetros otimizados pelo AG. Para a análise dos resultados, considera-se a diferença entre os tempos de vida 
simulados e os tempos de vida experimentais, obtida a partir do cálculo do erro relativo percentual.

A validação da estimação dos parâmetros é exposta na Figura 2, que contém a curva experimental pulsada utilizada na parametrização do modelo, ou seja, de $400 \mathrm{~mA}$; e a curva simulada gerada a partir da aplicação deste mesmo perfil de descarga pulsada ao modelo. Nota-se que o comportamento da curva simulada assemelha-se à curva experimental, estando ambas muito próximas. Neste caso, o $t_{v s}$ é de $12300 s$ e $t_{v e}$ é de $12338 s$, apresentando o erro significativamente baixo de $0,30 \%$.

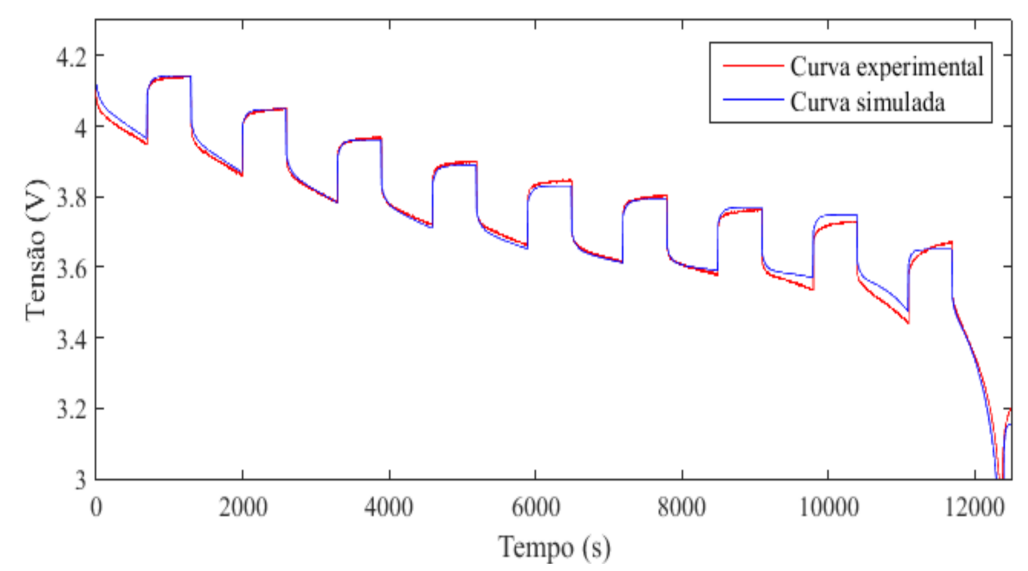

Figura 2: Validação da estimação de parâmetros.

O modelo é submetido a 4 diferentes perfis de descargas constantes a fim de validá-lo. $\mathrm{Na}$ Tabela 1 são apresentados estes perfis com seus respectivos tempos de vida simulados e experimentais médios, evidenciando os erros do modelo. O menor erro é obtido para o perfil de $500 \mathrm{~mA}$ devido a este se encontrar mais próximo da intensidade da corrente usada para estimar os parâmetros do modelo. Ao considerar as simulações realizadas para estes perfis, percebe-se que este modelo apresenta erros consideravelmente baixos, obtendo a média de $0,71 \%$.

Tabela 1: Validação do modelo.

\begin{tabular}{|c|c|c|c|}
\hline Perfis $(m A)$ & $t_{v s}(s)$ & $t_{v e}$ médio $(s)$ & Erro $(\%)$ \\
\hline 100 & 28030 & 27819,6 & 0,75 \\
\hline 250 & 11110 & 11244,8 & 1,19 \\
\hline 500 & 5495 & 5472,6 & 0,40 \\
\hline 650 & 4195 & 4217 & 0,52 \\
\hline
\end{tabular}




\section{Conclusão}

Neste trabalho foi realizada a estimação dos parâmetros do modelo para Predizer Runtime e Características V-I a partir da aplicação do método heurístico AG, sendo este modelo utilizado para a modelagem matemática do tempo de vida de baterias de LiPo. Assim como o AG proposto, o modelo também foi implementado na ferramenta computacional Matlab. A validação do modelo e do método utilizado para a parametrização foi feita por meio da análise comparativa entre os resultados simulados e os dados experimentais, adquiridos na plataforma de testes. Diante dos resultados obtidos, verifica-se que o modelo é capaz de predizer com acurácia o tempo de vida das baterias de LiPo, e que a utilização do AG sistematiza o processo de parametrização sem que este dependa de aspectos subjetivos, assim como ocorre na análise visual de curvas.

\section{Agradecimentos}

Os autores agradecem à Capes, pelo apoio financeiro e à Unijuí e o GAIC, pela infraestrutura.

\section{Referências}

[1] M. Chen and G. Rincón-Mora, Accurate electrical battery model capable of predicting runtime and i-v performance, IEEE Transactions on Energy Conversion, vol. 21, no. 2, pp. 504-511, Junho, 2006.

[2] G. P. Hammond, T. Hazeldine, Indicative energy technology assessment of advanced rechargeable batteries, Applied Energy, vol.138, pp. 559-571, 2015.

[3] J. H. Holland, Adaptation in Natural and Artifcial Systems, University of Michigan Press, Ann Arbor, MI, USA, 1975.

[4] T. Kim, W. Qiao, A hybrid battery model capable of capturing dynamic circuit characteristics an nonlinear capacity efects, IEEE Wireless Communications and Networking Conference, vol. 26, pp. 1172-1180, 2011.

[5] W. H. Meyer, Polymer Electrolytes for Lithium-Ion Batteries, Advanced Materials 10, pp. 439-448, 1998.

[6] D. Rakhmatov and S. Vrudhula, An analytical high-level battery model for use in energy management of portable electronic systems, National Science Foundation's State/Industry/University Cooperative Research Centers (NSFS/IUCRC), Center for Low Power Electronics (CLPE), pp. 1-6, 2001.

[7] V. Ramadesigan, P. W. C. Northrop, S. De, S. Santhanagopalan, R. D. Braatz, V. R. Subramanian, Modeling and simulation of lithium-ion batteries from a systems engineering perspective, Journal of The Electrochemical Society, vol. 159, pp. 31-45, 2012 . 